\title{
Persistent Penumbra in a Rabbit Stroke Model: Incidence and Histologic Characteristics
}

\author{
Leah J. Hennings, ${ }^{1}$ Rene Flores, ${ }^{2}$ Paula K. Roberson, ${ }^{3}$ Aliza Brown, ${ }^{2}$ John Lowery, ${ }^{4}$ \\ Michael Borrelli, ${ }^{2}$ and William C. Culp ${ }^{2}$ \\ ${ }^{1}$ Department of Pathology, College of Medicine, University of Arkansas for Medical Sciences, Little Rock, AR 72205, USA \\ ${ }^{2}$ Department of Radiology, College of Medicine, University of Arkansas for Medical Sciences, Little Rock, AR 72205, USA \\ ${ }^{3}$ Department of Biostatistics, College of Medicine, University of Arkansas for Medical Sciences, Little Rock, AR 72205, USA \\ ${ }^{4}$ Division of Laboratory Animal Medicine, College of Medicine, University of Arkansas for Medical Sciences, \\ Little Rock, AR 72205, USA
}

Correspondence should be addressed to Leah J. Hennings, lhennings@uams.edu

Received 31 October 2010; Revised 4 April 2011; Accepted 20 April 2011

Academic Editor: David S. Liebeskind

Copyright (c) 2011 Leah J. Hennings et al. This is an open access article distributed under the Creative Commons Attribution License, which permits unrestricted use, distribution, and reproduction in any medium, provided the original work is properly cited.

\begin{abstract}
Duration and extent of penumbra determine the window and brain volume in which interventions may save injured tissue after stroke. Understanding the penumbra in animals is necessary in order to design models that translate to effective clinical therapies. New Zealand white rabbits were embolized with aged autologous clot $(n=23)$ or insoluble microspheres $(n=21)$. To examine effects of treatment on penumbra, sphere-stroked animals were treated with $3 \mu \mathrm{m}$ microbubbles plus ultrasound $(n=19)$. Rabbits were euthanized at 4 or $24 \mathrm{hr}$. Infarct volume was measured following triphenyltetrazolium chloride (TTC) staining of brain sections. Penumbra was visualized using immunostaining of pimonidazole injected fifteen minutes prior to euthanasia. Potentially reversible penumbra was present in $14.3 \%$ stroked rabbits at 4 hours and $15.7 \%$ at 24 hours after embolic stroke and represented up to $35 \%$ of total lost tissue. Intervention at up to 24 hours may benefit a significant patient population.
\end{abstract}

\section{Introduction}

The degree of neurologic impairment following a stroke depends to a large extent on the volume of brain tissue lost and the specific anatomic structures involved. Following local ischemia, a core population of neurons in a region with extremely low cerebral blood flow is terminally injured within a relatively short period of time. Because average time to hospitalization for stroke is greater than 90 minutes [1], this central ischemic core cannot, in general, be saved. The region around the ischemic core with blood flow that is significantly reduced, but not below levels compatible with cell life, is extremely vulnerable to further insult from oxidative damage, hemorrhage, and inflammation during reperfusion. This sublethally injured penumbra, or area at risk, is eventually subsumed into the ischemic core, unless perfusion improves, and may comprise up to $70 \%$ of the total infarct volume. Stroke therapies that encourage survival of cells in this region result in better functional outcomes for patients.
Hypoxia within the penumbra may persist for 48 hours after stroke [2-5]. Whether or not injured cells in the penumbra progress to irreparable damage and cell death is dependent on small perfusion pressure change [6], and stroke therapy interventions to increase cerebral microvascular perfusion may have marked clinical impact through reduction of the amount of hypoxic penumbral tissue that progresses to cell death. Following reduced blood flow in stroke, cerebral microvessels undergo adaptations to increase perfusion, including active dilation to increase blood flow [7]. Other responses may be maladaptive for the penumbra, including the release of adhesion molecules and focal loss of blood-brain barrier to permit influx of inflammatory cells and fibrin $[8,9]$. Exposure to tissue factor with resultant thrombosis can occur where vascular flow is decreased, as in small vessels in the distribution bed of a large artery [9]. Ultrasound- (US-) facilitated thrombolysis with microbubbles (MBs) has been demonstrated to lyse clots in animal models with and without exogenous thrombolytics such as 
tissue plasminogen activator (tPA) [10-13]. Thrombolytic therapies may improve perfusion in small vessels, restoring cerebral blood flow to the penumbra even when recanalization of the blocked artery does not occur.

In acute human stroke, clinical imaging with CT perfusion studies or MRI diffusion studies provide some measures of penumbra which are of use in patient selection for therapy. Patients with visible penumbra may respond well to therapy hours beyond the standard temporal guidelines for thrombolytic treatment [14].

It is therefore of the utmost importance to determine the duration of the penumbra in preclinical models of stroke therapy, as this time represents the window of opportunity for intervention. Understanding possible differences in the development and duration of the penumbra in animal models is necessary in order to design meaningful animal models that translate to effective clinical therapies. Pimonidazole (Hypoxyprobe) has been demonstrated to accurately label hypoxic regions in the brain [15]. The objectives of this study were to examine the incidence and histologic features of penumbra in rabbits at 24 hours after infarction using Hypoxyprobe staining in two models of thromboembolic stroke and to determine effects of treatment with microbubbles and ultrasound on penumbra.

\section{Materials and Methods}

2.1. Preparation of Embolus. Emboli were prepared by obtaining an arterial blood sample $(2 \mathrm{~mL})$ from a donor rabbit. The blood was immediately transferred directly into a $30.5 \mathrm{~cm}$ length of Butterfly pediatric infusion set (no. 4506; Abbot Hospitals, Inc.; North Chicago, Ill, USA) tubing and allowed to clot at $37^{\circ} \mathrm{C}$ for 3 hours. Following incubation, but prior to embolization, the clot was expelled from the tubing into a dish containing physiological saline and subsequently cut into several pieces of $1.0 \mathrm{~mm}$ in length with an approximate diameter of $0.6 \mathrm{~mm}$. A single clot piece was drawn into a $3.0 \mathrm{~mL}$ syringe containing physiological saline for injection into the ICA with 0.7 to $2.0 \mathrm{~mL}$ of saline flush. Care was taken during each flush to ensure that no air bubbles were present in the syringe.

2.2. Animal Procedure. All animal procedures were approved by the Institutional Animal Care and Use Committee. The surgical and angiographic procedures were previously described by Brown et al. [16]. Briefly, male and female New Zealand white rabbits $(n=63$; mean body weight $=$ $5.2 \pm 0.07 \mathrm{~kg}$ ) were randomly assigned to stroke model groups and anesthetized. The right side of each rabbit's head was clipped, and depilatory cream was applied. A single plane C-arm digital subtraction angiography machine (OEC 9800; GE Healthcare; Salt Lake City, Utah, USA) was utilized for all angiographic procedures. Baseline subselective internal carotid artery (ICA) magnification angiography was performed via the right femoral artery in lateral and frontal projections to assess the cerebral vasculature of each rabbit. Rabbits were embolized by injection of $1.0 \mathrm{~mm}$ blood clot aged for 3 to 5 hours or 700-900 $\mu \mathrm{m}$ microspheres (Embosphere; BioSphere Medical Inc.; Rockland, Mass, USA) in
0.7 to $2.0 \mathrm{~mL}$ of physiologic saline into the ICA, occluding branches. One minute following embolization, repeat angiography was performed, and the degree and location of the arterial occlusion were documented.

For animals in the treatment group, an intravenous catheter (Instyle-W; Becton Dickinson; Sandy, Utah, USA) placed into the left ear vein was used for administration of $3 \mu \mathrm{m} \mathrm{MB}$, and transcutaneous pulsed wave (20\% duty cycle) US at $1 \mathrm{MHz}$ and $0.8 \mathrm{~W} / \mathrm{cm}^{2}$ (Sonicator 716; Mettler Electronics; Anaheim, Calif, USA) was applied for one hour. Following embolization a hand-held $10 \mathrm{~cm}^{2}$ therapeutic transducer was placed in front of the ear and behind the eye and was coupled to the skin with standard US gel. Positioning of the US probe was confirmed fluoroscopically.

Rabbits exhibiting a complex origin of the internal carotid artery preventing subselection and subsequent embolization on initial angiography $(n=3)$ and rabbits that did not undergo angiography $(n=4)$ were used as unstroked controls for pimonidazole staining. Animals were euthanized via IV pentobarbital overdose at 4 or 24 hours after embolization.

2.3. Measurement of Infarct Volume. Brain was harvested immediately after euthanasia, chilled in physiologic saline on melting ice for 60 minutes, and sliced coronally at $0.4 \mathrm{~cm}$ intervals using a mold and slicing guide (Harvard Apparatus) to assure consistent sectioning. All coronal brain sections were placed in 1\% 2,3,5-triphenyltetrazolium chloride (Sigma-Aldrich; St. Louis, Mo, USA) for 45 minutes at $37^{\circ} \mathrm{C}$, fixed in $10 \%$ formalin, and photographed with a digital camera. Section area and area of infarction were measured using $\mathrm{NIH}$ ImageJ. Infarct and section volumes for each slice were calculated by multiplying each value by the slice thickness of $4 \mathrm{~mm}$. Total brain percent infarct volume was calculated by dividing the sum of the infarct volumes into the sum of the section volumes and multiplying by $100 \%$.

2.4. Histopathology. To verify infarct locations each brain section was fixed in 10\% formalin, processed and embedded into paraffin, sectioned at $4 \mu \mathrm{m}$, and stained with hematoxylin and eosin. Each brain coronal section was evaluated by a veterinary pathologist without knowledge of treatment group.

Hypoxia in the penumbra may be evaluated using nitroimidazole compounds that are reduced in living cells to a radical anion. In hypoxic cells, this compound irreversibly binds cellular components and accumulates [16]. The compound does not bind in cells that are not hypoxic, and it is not metabolized by dead cells in infarcted areas. All rabbits received $100 \mathrm{mg}$ of pimonidazole (Chemicon) IV 15 minutes prior to euthanasia. Following TTC staining, formalin fixation, and processing as described above, all sections from each brain were immunolabeled using an antibody specific for pimonidazole (Hypoxyprobe, Chemicon). Briefly, sections were deparaffinized and antigen retrieval was performed via decloaking in tris-buffered saline tween-20 (TBST) for 20 minutes. Dako peroxidase block was applied for 10 minutes, and sections were rinsed three times in TBST. 
TABLE 1: Incidence of penumbra.

\begin{tabular}{llccc}
\hline Model & Group & Number & Time point & $\begin{array}{c}\text { Incidence of } \\
\text { penumbra }\end{array}$ \\
\hline Sphere & $3 \mu \mathrm{m} \mathrm{MB}+\mathrm{US}$ & 19 & 24 & $4 / 14(28.6 \%)$ \\
Sphere & Control & 14 & 24 & $2 / 14(14 / 3 \%)$ \\
Clot & Control & 23 & 24 & $2 / 23(8.7 \%)$ \\
Sphere & Control & 7 & 4 & $1 / 7(14.3 \%)$ \\
\hline
\end{tabular}

Summary of penumbra incidence by group. No significant differences in incidence were noted by model $(P=0.62)$, time point $(P=1.0)$, or treatment $(P=0.65)$.

$10 \%$ normal goat serum was applied for 30 minutes, and sections were blotted. Primary monoclonal antibody to bound pimonidazole (Chemicon) was applied at a 1:50 dilution in Dako diluent with background blocking agents for 40 minutes. Sections were rinsed three times in TBST, and secondary Vector antigoat IgG antibody (Vector) diluted at $1: 400$ in Dako diluent with background blocking agents was applied for 30 minutes. Sections were rinsed three times in TBST. Dako DAB+ was applied for 3 minutes. Sections were rinsed in TBST and lightly counterstained with hematoxylin. Brain sections from animals that did not receive pimonidazole as well as sections stained without primary antibody are used as negative controls.

Hypoxyprobe- stained slides were digitally scanned using the Aperio ScanScope T2 (Aperio, Vista, Calif, USA). The Scanscope produces high resolution slides which may be viewed and analyzed at magnifications up to 200x. Scanned slides were examined to detect penumbral staining. Penumbra was defined as strong staining (within the upper $90 \%$ of staining intensity) immediately adjacent to and contiguous with regions of necrosis. Sections were analyzed using morphometrics (ImageScope, Aperio) for area of penumbra, area of infarct, and area of penumbra plus infarct. Lesion size was measured as the area of the penumbra and the associated infarct. Penumbral area was measured, and relative size was given as the percent of total lesion. When multiple infarcted regions were present within a section, areas for penumbra and infarcts were summed to give a total area.

2.5. Statistical Analysis. Incidence of penumbra among groups was compared using Fisher's exact test as implemented in the statistical software package StatXact. The relationship between penumbra size and percent infarct volume was assessed using both Pearson and Spearman correlation coefficients, because of the potential for a nonlinear association. A $t$-test was used to compare the mean percent stroke volumes between those rabbits with and without penumbra. Stroke volumes are presented as mean \pm standard deviation.

\section{Results}

Repeat angiography (Figures 1(a) and 1(b)) demonstrated occlusion in 56 animals. Location of occlusion and numbers of animals affected are given in the table of Figure 1. The most common type of occlusion on repeat angiography was single occlusion in the middle cerebral artery (MCA, 26/63).
Additional 18 animals had occlusion of both the ACA and the middle cerebral artery (MCA). Triple occlusions involving the cerebral arteries occurred in 3 animals, and the occipital artery $(\mathrm{OA})$ was occluded in one animal. No visible occlusion was noted in 7/63 animals, all from the clot control group, although measurable stroke volume was noted with TTC stain for these animals, and all had histologic infarction. Clot model was also associated with fewer MCA and ACA occlusions $(P<0.001, P<0.005$, resp.). No other significant differences were noted for occlusion location and model or treatment group.

Infarct volume in stroked animals ranged from $0.1 \%$ to $18.4 \%$. Unstroked staining control rabbits had stroke volume of 0 , as expected. Penumbra was evident in Hypoxyprobestained sections as intense staining adjacent to and contiguous with infarct (Figure 2). Brain sections from all animals exhibited diffuse mild hypoxia as demonstrated by weak to moderate cellular pimonidazole staining, and necrotic tissue (infarct) was not labeled by pimonidazole (Figure 2(c)). No labeling was observed in control sections from rabbits which were not given pimonidazole (Figure 2(d)) and sections stained without primary antibody (data not shown). Penumbra was observed in $1 / 7$ rabbits $(14.3 \%)$ at 4 hours and in $8 / 51(15.7 \%)$ rabbits at $24 \mathrm{~h}$ (Table 1$)$.

Incidence of penumbra did not differ with stroke model $(P=0.62$; clot control versus sphere control) or with treatment at 24 hours $(P=0.65$; treated versus sphere control, Table 1). Penumbral staining occurred around both large and small infarcts, and penumbra size was not significantly correlated with percent infarct volume $(P>0.29)$ at 24 hours. Mean stroke volume percent did not differ between rabbits with or without penumbra at 24 hours $(4.43 \pm$ 4.97 versus $3.60 \pm 4.56$, resp.; $P=0.64$ ). The average size of penumbra as a percentage of total lesion (infarct plus penumbra) at 24 hours was $14 \% \pm 4 \%$. Penumbra ranged from $0.96 \%$ to $35 \%$ of the total lesion. Rarely, hypoxic tissue encircled the ischemic core, but penumbra was more often eccentric, encompassing only a portion of the circumference of the ischemic core.

\section{Discussion}

Preclinical animal models are an important tool in evaluation of potential stroke therapies. It is of vital importance to understand the pathogenesis of brain loss in these models and to determine what the optimal window of treatment therapy is for each model as well as the optimal time for evaluation of cerebral damage. As stroke develops, hypoxic tissue peripheral to the ischemic core (penumbra) undergoes progressive damage culminating in cell death if perfusion does not improve. This tissue represents the area of the brain most amenable to stroke therapies and most vulnerable to reperfusion injury. The volume of the penumbra may be equal to or greater than the volume of the ischemic core on CT perfusions or MRI diffusion imaging [17]; therefore, treatments that protect this vulnerable region from further injury may result in significantly reduced final stroke volume and improved clinical outcome. 


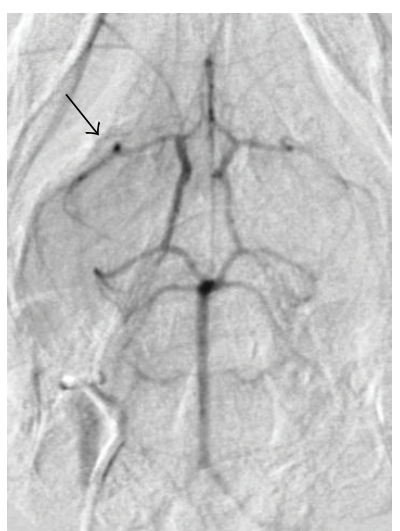

(a)

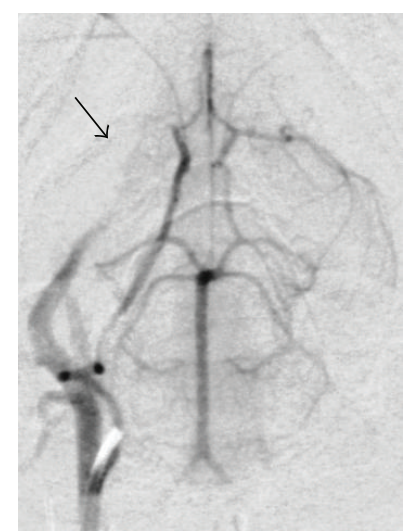

(b)

\begin{tabular}{|l|c|}
\hline Location & Number \\
\hline Middle cerebral artery & 27 \\
\hline Occipital artery & 1 \\
\hline MCA+anterior cerebral artery (ACA) & 23 \\
\hline MCA+posterior cerebral artery (PCA) & 1 \\
\hline MCA+PCA+superior cerebellar artery (SCA) & 1 \\
\hline MCA+ACA+PCA & 3 \\
\hline No visible occlusion & 7 \\
\hline
\end{tabular}

FIGURE 1: Angiography results. Pre-embolization angiogram (a) demonstrates filling of the MCA. Occlusion and absence of filling are evident in postembolization angiogram (b). Arrows indicate location of the MCA. Location of occlusion and numbers of animals affected by each type are given in the table.

In contrast to studies to determine the optimal window of therapy, studies designed to examine cerebral injury and effects of treatment on total stroke volume should be evaluated at a time point when hypoxic penumbra is no longer evident and stroke is maximally developed. For these reasons, it is vitally important to determine the duration of penumbra in preclinical animal models. Although penumbra has been documented in humans at 48 hours after infarction $[2,3]$, no penumbra was evident in a rat model at 48 hours $[18,19]$. Using in vivo PET imaging of F-fluoromisonidazole in a rat carotid artery occlusion model, Takasawa et al. [18] demonstrated that significant penumbra is not present at 48 hours after occlusion. However, this study did not examine time points between 180 minutes and 48 hours. Saita et al. [4] found negligible uptake of F-fluoromisonidazole in 4 rats at 22 hours after infarction. Noto et al. [21] used a similar compound, pimonidazole (Hypoxyprobe, Chemicon), to evaluate the penumbra in a rat model of focal cerebral ischemia and found increased Hypoxyprobe labeling in 5/5 animals at 24 hours after infarction $[20,21]$. The objective of this study was to determine the incidence of hypoxic penumbra in two rabbit thromboembolic stroke models and to examine the effects of thrombolytic therapy on penumbra at 24 hours after infarction.

Penumbra was easily distinguished as clearly defined regions of very intense staining adjacent to and contiguous with infarcts. This staining pattern was observed in $15.7 \%$ of animals at 24 hours. There is convincing evidence that the penumbra is likely to succumb to cell death over time and therefore contribute up to $50 \%$ of the total brain loss in stroke $[6,19]$. In the rabbits of this study, the penumbra represented on average approximately $14 \%$ of the total lesion. This result is consistent with previously published findings in humans and rats $[19,22]$. Penumbra was occasionally circumferential but more often did not completely encircle infarct. This pattern is consistent with reperfusion gradients that depend on available collateral blood supply. The penumbra was very similar in size and shape to penumbra imaged using ${ }^{18} \mathrm{~F}$ fluoromisonidazole PET [18], a compound that accumulates in hypoxic tissue using the same mechanism as pimonidazole, and 2-deoxyglucose PET [19] in rats. Despite some differences in location of occlusion between groups on postembolization angiography, no significant differences were found in incidence of penumbra. This suggests that persistence of penumbra is independent of type of occlusion.

The observed differences in location of occlusion between clot and sphere models may be explained by functional differences in type of embolus It is of particular interest that all animals without visible occlusion in major arteries but histologic evidence of stroke were embolized with aged clot. This suggests that clot may be partially lysed by endogenous processes during and immediately following embolization, at least in some animals, resulting in fragmentation and blockage of smaller vessels downstream. 


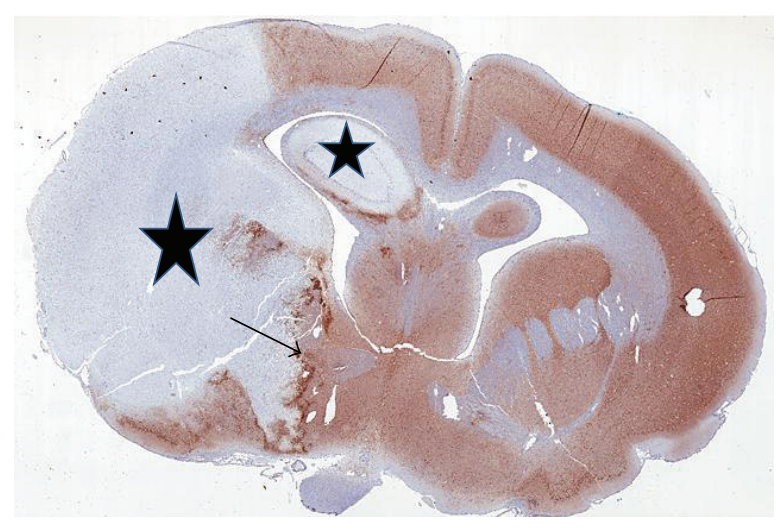

(a)

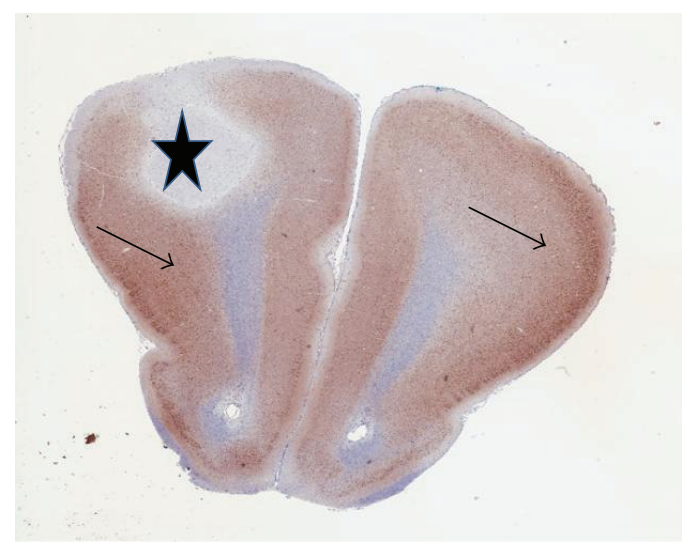

(c)

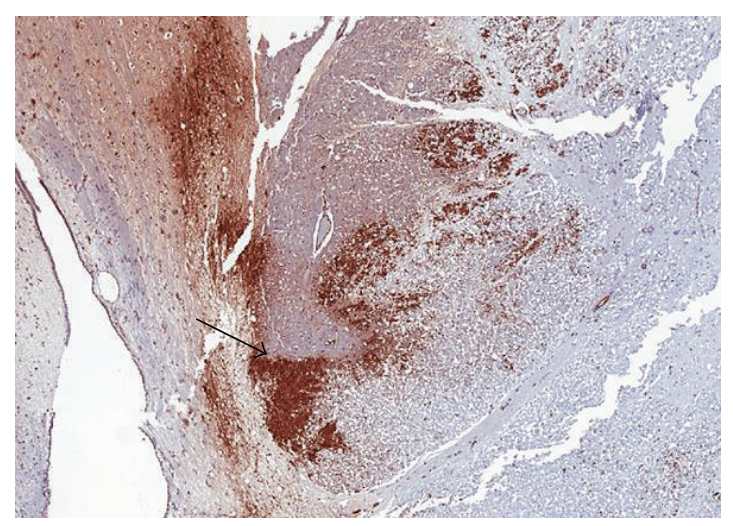

(b)

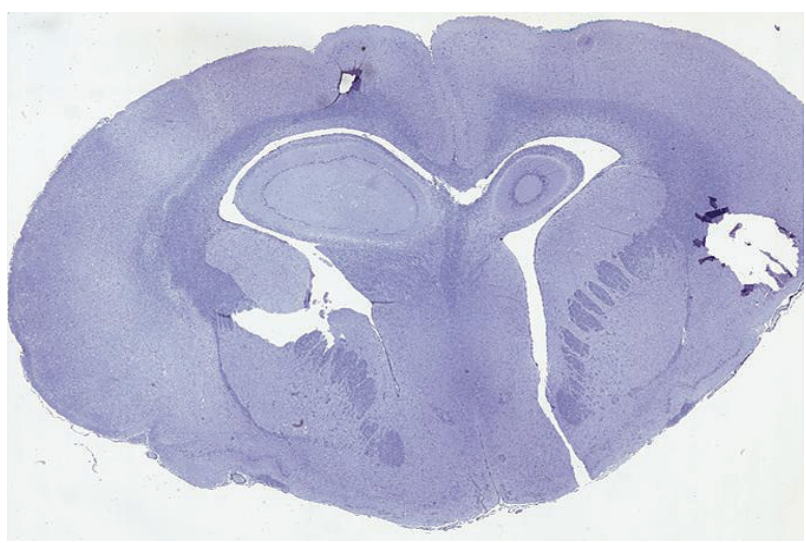

(d)

Figure 2: Pimonidazole stain identifies penumbra. (a) Intense staining is present in penumbra (arrow) adjacent to infarct (stars), $0.5 \times$ magnification. (b) Closeup of area within the box demonstrates intense staining adjacent to infarct, $4 \times$ magnification. (c) Diffuse weak staining (arrows) indicates mild hypoxia associated with euthanasia. Infarct does not stain (star.), $0.5 \times$ magnification. Negative control (d).

Although penumbra persisted for 24 hours in some animals, no effect of treatment with $3 \mu \mathrm{m}$ microbubbles and ultrasound on the incidence of penumbra was noted. This indicates that this treatment exerts protective effects, if any, on penumbra at early time points. However, other treatments may have more significant effect on penumbra, and further study is warranted. Currently in the United States, approximately $3 \%$ of strokes are treated using IV thrombolytic therapy applying a 4.5-hour window or using intra- arterial clot removal applying a 6-hour window [23]. With accurate penumbra imaging, much later interventions may be successfully applied [14]. In this rabbit model $15.7 \%$ had significant penumbra, potentially curable areas of brain damage even at 24 hours. This suggests that clinical tests for penumbra, CT perfusion, or MRI diffusion studies should be performed regardless of stroke duration to select these patients who can still profit from intervention at much later times than currently is the standard of care [24-26]. Our results suggest that therapy could potentially benefit a much greater patient population.

Diffuse mild cerebral hypoxia, characterized by weak to moderate, diffuse staining with pimonidazole, was evident in all animals. This staining was not present in control sec- tions from animals not given pimonidazole or controls stained without the primary antibody, and it most likely represents binding of pimonidazole in hypoxic brain induced by the euthanasia process, which involves sedation followed by intravenous injection of pentobarbital. To avoid mischaracterization of this background hypoxia as penumbra, threshold for penumbra was set at the upper $90 \%$ of staining intensity and included only staining contiguous with infarcts. Certainly brain swelling can compromise blood flow in areas away from the primary infarct. This may have excluded some areas of actual penumbra by generally underestimating its volume or presence.

Hypoxic penumbra remained in $15.7 \%$ of rabbits at 24 hours. This suggests that a significant number of patients may benefit from additional imaging at 24 hours, with continued intervention if residual penumbra is present.

\section{Acknowledgments}

This work was supported by NIH Grant 1R01HL08248101 for W. C. Culp (PI). The authors wish to thank Jennifer James and Shirley Mullins of the Experimental Pathology Core Laboratory, UAMS, for histology services. 


\section{References}

[1] M. J. Alberts, C. Bertels, and D. V. Dawson, "An analysis of time presentation after stroke," Journal of the American Medical Association, vol. 263, no. 1, pp. 65-68, 1990.

[2] S. J. Read, T. Hirano, D. F. Abbott et al., "The fate of hypoxic tissue on $18 \mathrm{~F}$-fluoromisonidazole positron emission tomography after ischemic stroke," Annals of Neurology, vol. 48, no. 2, pp. 228-235, 2000.

[3] S. J. Read, T. Hirano, D. F. Abbott et al., "Identifying hypoxic tissue after acute ischemic stroke using PET and F-fluoromisonidazole," Neurology, vol. 51, no. 6, pp. 1617-1621, 1998.

[4] K. Saita, M. Chen, N. J. Spratt et al., "Imaging the ischemic penumbra with $18 \mathrm{~F}$-fluoromisonidazole in a rat model of ischemic stroke," Stroke, vol. 35, no. 4, pp. 975-980, 2004.

[5] W. D. Heiss, L. Kracht, M. Grond et al., "Early [(11)C]flumazenil/HO positron emission tomography predicts irreversible ischemic cortical damage in stroke patients receiving acute thrombolytic therapy," Stroke, vol. 31, no. 2, pp. 366369,2000 .

[6] M. D. Ginsberg, "Adventures in the pathophysiology of brain ischemia: penumbra, gene expression, neuroprotection: the 2002 Thomas Willis lecture," Stroke, vol. 34, no. 1, pp. 214 223, 2003.

[7] A. Y. Shih, B. Friedman, P. J. Drew, P. S. Tsai, P. D. Lyden, and D. Kleinfeld, "Active dilation of penetrating arterioles restores red blood cell flux to penumbral neocortex after focal stroke," Journal of Cerebral Blood Flow and Metabolism, vol. 29, no. 4, pp. 738-751, 2009.

[8] G. J. del Zoppo and T. Mabuchi, "Cerebral microvessel responses to focal ischemia," Journal of Cerebral Blood Flow and Metabolism, vol. 23, no. 8, pp. 879-894, 2003.

[9] G. J. del Zoppo, "Virchow's triad: the vascular basis of cerebral injury," Reviews in Neurological Diseases, vol. 5, supplement 1, pp. S12-S21, 2008.

[10] G. Tsivgoulis, W. C. Culp, and A. V. Alexandrov, "Ultrasound enhanced thrombolysis in acute arterial ischemia," Ultrasonics, vol. 48, no. 4, pp. 303-311, 2008.

[11] S. C. Chen, J. L. Ruan, P. O. W. Cheng, Y. H. Chuang, and P. C. $\mathrm{Li}$, "In vitro evaluation of ultrasound-assisted thrombolysis using a targeted ultrasound contrast agent," Ultrason Imaging, vol. 31, no. 4, pp. 235-246, 2009.

[12] C. A. Molina, M. Ribo, M. Rubiera et al., "Microbubble administration accelerates clot lysis during continuous 2$\mathrm{MHz}$ ultrasound monitoring in stroke patients treated with intravenous tissue plasminogen activator," Stroke, vol. 37, no. 2, pp. 425-429, 2006.

[13] W. C. Culp, T. R. Porter, J. Lowery, F. Xie, P. K. Roberson, and L. Marky, "Intracranial clot lysis with intravenous microbubbles and transcranial ultrasound in swine," Stroke, vol. 35, no. 10, pp. 2407-2411, 2004.

[14] A. Abou-Chebl, "Endovascular treatment of acute ischemic stroke may be safely performed with no time window limit in appropriately selected patients," Stroke, vol. 41, no. 9, pp. 1996-2000, 2010.

[15] P. Thored, J. Wood, A. Arvidsson, J. Cammenga, Z. Kokaia, and O. Lindvall, "Long-term neuroblast migration along blood vessels in an area with transient angiogenesis and increased vascularization after stroke," Stroke, vol. 38, no. 11, pp. 3032-3039, 2007.

[16] A. T. Brown, R. D. Skinner, R. Flores et al., "Stroke location and brain function in an embolic rabbit stroke model," Journal of Vascular and Interventional Radiology, vol. 21, no. 6, pp. 903-909, 2010.
[17] L. Belayev, W. Zhao, R. Busto, and M. D. Ginsberg, "Transient middle cerebral artery occlusion by intraluminal suture: I. Three-dimensional autoradiographic image-analysis of local cerebral glucose metabolism blood flow interrelationships during ischemia and early recirculation," Journal of Cerebral Blood Flow and Metabolism, vol. 17, no. 12, pp. 1266-1280, 1997.

[18] M. Takasawa, J. S. Beech, T. D. Fryer et al., "Imaging of brain hypoxia in permanent and temporary middle cerebral artery occlusion in the rat using $18 \mathrm{~F}$-fluoromisonidazole and positron emission tomography: a pilot study," Journal of Cerebral Blood Flow and Metabolism, vol. 27, no. 4, pp. 679689, 2007.

[19] M. D. Ginsberg, L. Belayev, W. Zhao, P. W. Huh, and R. Busto, "The acute ischemic penumbra: topography, life span, and therapeutic response," Acta Neurochirurgica, vol. 1999, no. 73, supplement, pp. 45-50, 1999.

[20] T. Noto, Y. Furuichi, M. Ishiye et al., "Tacrolimus (FK506) limits accumulation of granulocytes and platelets and protects against brain damage after transient focal cerebral ischemia in rat," Biological and Pharmaceutical Bulletin, vol. 30, no. 2, pp. 313-317, 2007.

[21] T. Noto, Y. Furuichi, M. Ishiye et al., "Temporal and topographic profiles of tissue hypoxia following transient focal cerebral ischemia in rats," Journal of Veterinary Medical Science, vol. 68, no. 8, pp. 803-807, 2006.

[22] W. Zhao, L. Belayev, and M. D. Ginsberg, "Transient middle cerebral artery occlusion by intraluminal suture: 2 . neurological deficits, and pixel-based correlation of histopathology with local blood flow and glucose utilization," Journal of Cerebral Blood Flow and Metabolism, vol. 17, no. 12, pp. 1281-1290, 1997.

[23] D. Lloyd-Jones, R. J. Adams, T. M. Brown et al., "Heart disease and stroke statistics-2010 update: a report from the american heart association," Circulation, vol. 121, no. 7, pp. e46-e215, 2010.

[24] D. Kleindorfer, Y. Xu, C. J. Moomaw, P. Khatri, O. Adeoye, and R. Hornung, "US geographic distribution of rt-PA utilization by hospital for acute ischemic stroke," Stroke, vol. 40, no. 11, pp. 3580-3584, 2009.

[25] N. Ahmed, N. Wahlgren, M. Grond et al., "Implementation and outcome of thrombolysis with alteplase 3-4.5 h after an acute stroke: an updated analysis from SITS-ISTR," The Lancet Neurology, vol. 9, no. 9, pp. 866-874, 2010.

[26] W. Hacke, M. Kaste, E. Bluhmki et al., "Thrombolysis with alteplase 3 to 4.5 hours after acute ischemic stroke," New England Journal of Medicine, vol. 359, no. 13, pp. 1317-1329, 2008. 


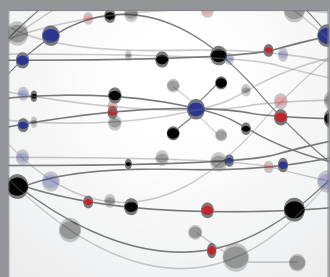

The Scientific World Journal
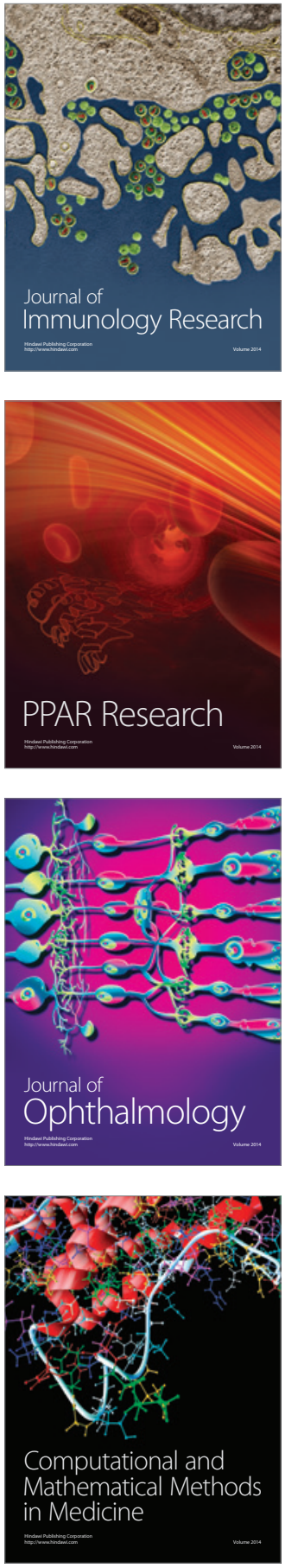

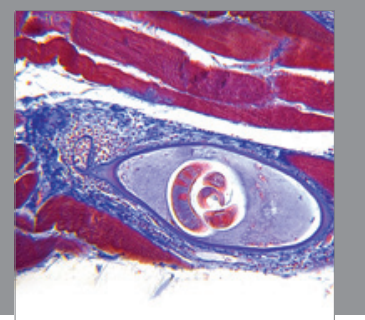

Gastroenterology

Research and Practice
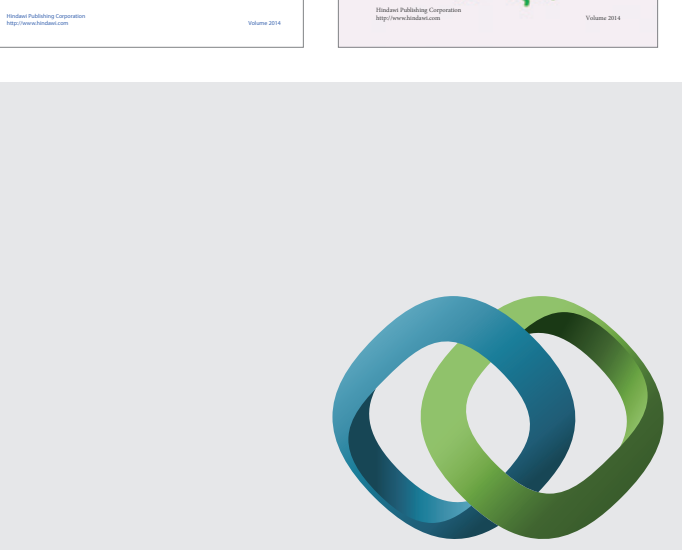

\section{Hindawi}

Submit your manuscripts at

http://www.hindawi.com
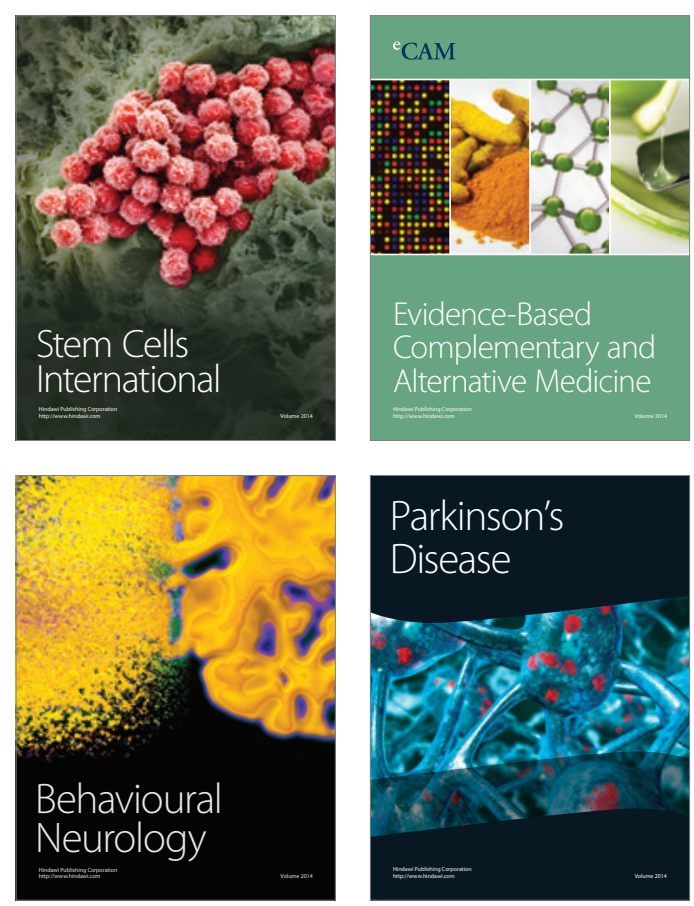

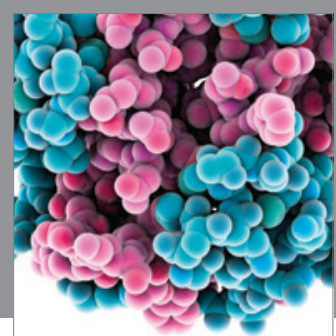

Journal of
Diabetes Research

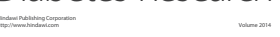

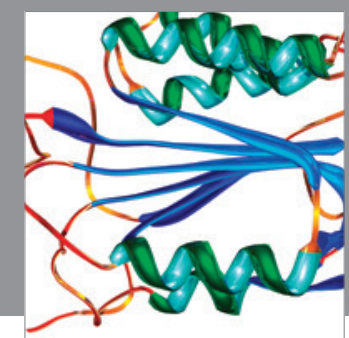

Disease Markers
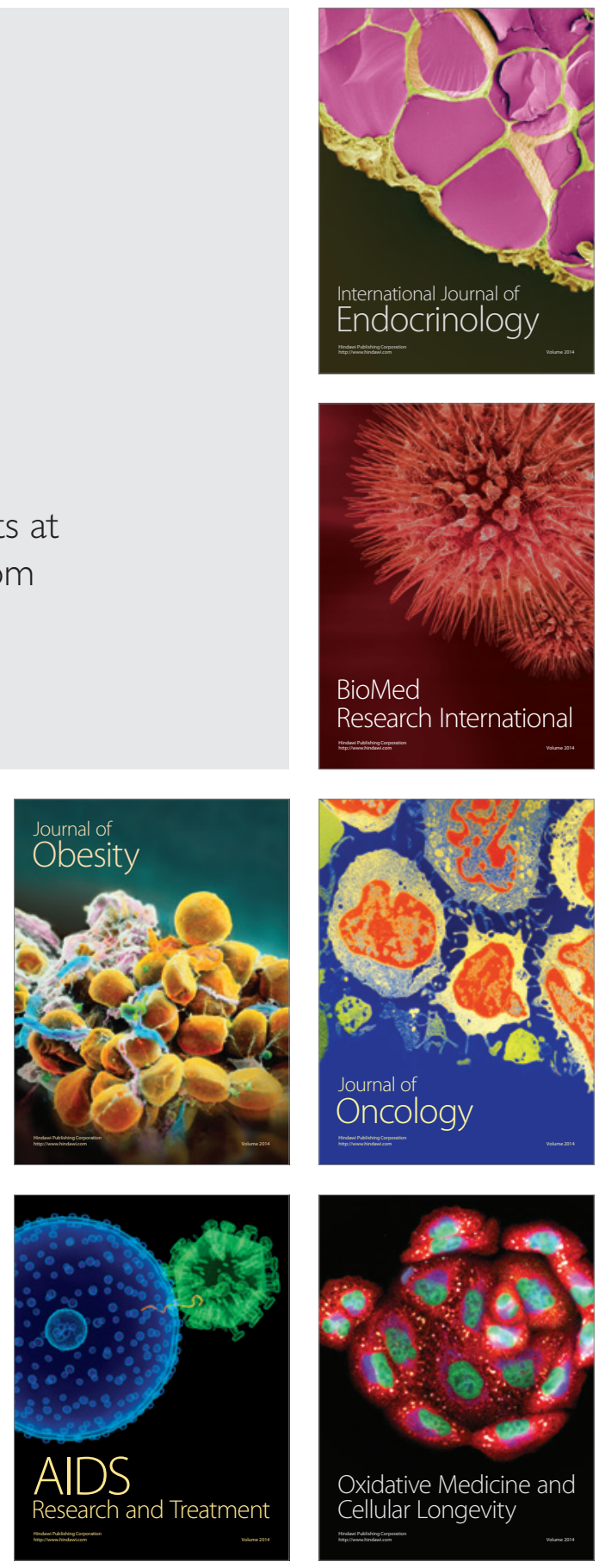